\title{
Spatio-temporal Brusselator Model and Biological Pattern Formation
}

\author{
Zakir Hossine $^{1}$, Oishi Khanam ${ }^{1}$, Md. Mashih Ibn Yasin Adan ${ }^{2}$ \\ and Md. Kamrujjaman ${ }^{2,3^{*}}$ \\ ${ }^{1}$ Department of Applied Mathematics, University of Dhaka, Dhaka 1000, Bangladesh. \\ ${ }^{2}$ Department of Mathematics, University of Dhaka, Dhaka 1000, Bangladesh. \\ ${ }^{3}$ Department of Mathematics and Statistics, University of Calgary, Calgary, AB, Canada.
}

Authors' contributions

This work was carried out in collaboration among all authors. Authors $\mathrm{ZH}, \mathrm{MK}$ and OK contributed to the study conceptualization, planned the study and coordinated the manuscript. Authors MK and ZH proposed the mathematical model and carried out the mathematical and numerical study. Authors MMIYA and OK drafted the manuscript writing. Authors MMIYA and ZH reviewed and author MK finalized the manuscript. All the authors accepted the finalized version of the manuscript.

Article Information

DOI: 10.9734/ARRB/2021/v36i530380

Editor(s):

(1) Prof. Ibrahim Farah, Jackson State University, USA.

Reviewers:

(1) Anthony J. Duben, USA.

(2) Oleg Ivanov, NRC Kurchatov Institute, Russia.

Complete Peer review History: http://www.sdiarticle4.com/review-history/69331

Original Research Article

Received 27 March 2021

Accepted 05 June 2021

Published 08 June 2021

\section{ABSTRACT}

This paper explores a two-species non-homogeneous reaction-diffusion model for the study of pattern formation with the Brusselator model. We scrutinize the pattern formation with initial conditions and Neumann boundary conditions in a spatially heterogeneous environment. In the whole investigation, we assume the case for random diffusion strategy. The dynamics of model behaviors show that the nature of pattern formation with varying parameters and initial conditions thoroughly. The model also studies in the absence of diffusion terms. The theoretical and numerical observations explain pattern formation using the reaction-diffusion model in both one and two dimensions.

${ }^{*}$ Corresponding author: E-mail: kamrujjaman@du.ac.bd; 
Keywords: Pattern formation; turing pattern; reaction-diffusion; numerical analysis.

2010 Mathematics Subject Classification: 92D25, 35K57 (primary), 35K61, 37N25.

\section{INTRODUCTION}

The branch of biology and medicine concerned with the study of embryos is known as embryology. Cell division is a crucial part of embryology; moreover, cell division ascends to the embryology study. The most concerning issue is how to cell embody and how to thrive the sequential formation. We consider the procedure that can yield pattern formation and propose a likely structure of spatial patterns to investigate this.

Pattern formation is seen in various sectors in living life. The pattern composition varies from individuals to individuals. The study of pattern formation shows complications of cell embody, which are altering with space and time. The genes rules pattern composition as well as improving tissues and organs in appropriates place within the body. In biology, self-evident is crucial part in pattern formation [1, 2, 3, 4, 5].

Turing pattern seems in the spatial system of nature identically or indiscriminately. Pattern formation observes animate objects and inanimate objects, for example, the surface of sand or water, which looks like a wave and periodic pattern formation. The leaf veins also look like periodic pattern formation; further, the cell of humans or animals can easily see pattern composition. Alan Turing did the first investigation, and he transformed pattern formation mathematically [3, 6, 7]. Turing considered two systems for generating patterns. One of them was a non-mixed system. This stationary state would be driven unstable by diffusion, and the other was a well-mixed system. There would be a spatially uniform stationary state that was stable to perturbations. The reaction-diffusion equation forms the pattern, and the general form of the equation is

$$
\frac{\partial u(t, x)}{\partial t}=f(u(t, x))+\mu \Delta u(t, x)
$$

where, $u(t, x)$ represents vector of chemical concentrations, $f(u(t, x))$ means the reaction kinetics; generally nonlinear and $\mu$ be a matrix of unchangeable diffusion coefficients (commonly diagonal). Moreover, the boundary and initial conditions, which are usually periodic and perturbations of the homogeneous stationary state, respectively, are used in the closure system $[1,4,8]$.

There are many kinds of models based on pattern formation. The Brusselator model is also such a model; mainly, this model is applied in the chemical reactions involved with trimolecular steps. Using reaction-diffusion systems in the Brusselator model would be more challenging $[2,4,6,8,9]$. The reaction-diffusion for the Brusselator model is

$$
\left\{\begin{array}{l}
u_{t}=\mu_{1} \Delta u(t, x)+\alpha-(\beta+1) u(t, x)+u^{2}(t, x) v(t, x), t>0, x \in \Omega, \\
v_{t}=\mu_{2} \Delta v(t, x)+\beta u(t, x)-u^{2}(t, x) v(t, x), t>0, x \in \Omega \\
\nabla u \cdot n=\nabla v \cdot n=0, t>0, x \in \partial \Omega \\
u(0, x)=u_{0}(x), v(0, x)=v_{0}(x), x \in \Omega
\end{array}\right.
$$

where $u(t, x)$ and $v(t, x)$ represent the population densities of two competing species which are non-negative. The constants $\mu_{1}$ and $\mu_{2}$ are the corresponding migration rates. The reaction kinetics functions are $f(t, x, u, v)=\alpha-(\beta+1) u(t, x)+u^{2}(t, x) v(t, x)$ and $g(t, x, u, v)=\beta u(t, x)-u^{2}(t, x) v(t, x)$.

In this paper, we are interested in analyzing the behavior of the model (1.1) in 2D coordinates. We have various spot or stripe patterns depending on the parameter's values and initial values. The main findings of this paper are to illustrate qualitative testimony in backing of a reaction-diffusion system for pattern formation, which is also analogous with living life and inert objects. The analytic results and 
numerical illustrations present different types of natural patterns by the Brusselator model; at least three are visible in this study.

\section{STABILITY ANALYSIS: CLASSICAL REACTION-DIFFUSION EQUATIONS}

In this section, we discuss the stability of reaction-diffusion equations. Typically during the reactiondiffusion system is non-linear. It is not easy to realize how a particular solution will be uplifted over space and time. Nevertheless, a particular result over time gives some idea about the nature of the solution. For stability analysis, we need to linearize the reaction function near the homogeneous stationary state solution. The specific steady-state solution over time gives a notion about the convergence of zero for which growth rates. Moreover, we get translucent conception about instability for several conditions. We consider one-two spatial dimensions for our study.

We want to understand the formation of spatial patterns on an animal's skin. During the embryo's growth, we have chemical reactions, and cell proliferation can gradients in concentration arise in the presence of diffusion and reaction. In the absence of diffusion, the results can be found in Appendix A.

In generalized form, the resulting system of differential equations is

$$
\left\{\begin{array}{l}
\frac{\partial u}{\partial t}=\mu_{1} \Delta u(t, x)+f(t, x, u, v), t>0, x \in \Omega \\
\frac{\partial v}{\partial t}=\mu_{2} \Delta v(t, x)+g(t, x, u, v), t>0, x \in \Omega \\
\frac{\partial u}{\partial n}=\frac{\partial v}{\partial n}=0 \\
u(0, x)=u_{0}(x), v(0, x)=v_{0}(x), \quad x \in \Omega
\end{array}\right.
$$

where, $\mu_{1}$ and $\mu_{2}$ are different, to get flexibility. In the $X$-direction we have an infinite extent. If we have finite extent in $X$-direction, species can not leave, $\frac{\partial u}{\partial n}=0, \frac{\partial v}{\partial n}=0$ are the no flux boundary conditions [5]. Let us assume that $u^{*}$ and $v^{*}$ are the concentrations of the reaction-diffusion system at the stationary state. We linearize the reaction-diffusion model by letting $\widetilde{u}=u-u^{*}$ and $\widetilde{v}=v-v^{*}$. Therefore, from the reaction-diffusion model, we get

$$
\begin{aligned}
& \frac{\partial \widetilde{u}}{\partial t}=\mu_{1} \Delta \widetilde{u}+f_{u} \widetilde{u}+f_{v} \widetilde{v}, \\
& \frac{\partial \widetilde{v}}{\partial t}=\mu_{2} \Delta \widetilde{v}+g_{u} \widetilde{u}+g_{v} \widetilde{v},
\end{aligned}
$$

which is the modified reaction-diffusion equation. Now, the solution of this system is

$$
\begin{aligned}
& \widetilde{u}(t, x)=\dot{u} e^{\sigma t} \sin \gamma x \\
& \widetilde{v}(t, x)=\dot{v} e^{\sigma t} \sin \gamma x,
\end{aligned}
$$

where $\dot{u}$ and $\dot{v}$ be the non-zero stationary state. So, from the modified reaction-diffusion equation

$$
\sigma e^{\sigma t} \dot{u} \sin \gamma x=-\gamma^{2} \mu_{1} \dot{u} e^{\sigma t} \sin \gamma x+f_{u} \dot{u} e^{\sigma t} \sin \gamma x+f_{v} \dot{v} e^{\sigma t} \sin \gamma x .
$$

Dividing both sides by $e^{\sigma t} \sin \gamma x$, we get

$$
\sigma \dot{u}=-\gamma^{2} \mu_{1} \dot{u}+f_{u} \dot{u}+f_{v} \dot{v} .
$$

Analogously, we obtain

$$
\sigma \dot{v}=-\gamma^{2} \mu_{2} \dot{v}+g_{u} \dot{u}+g_{v} \dot{v}
$$


Now, let us write this in vector form

$$
\sigma\left[\begin{array}{c}
\dot{u} \\
\dot{v}
\end{array}\right]=\left[\begin{array}{cc}
f_{u}-\gamma^{2} \mu_{1} & f_{v} \\
g_{u} & g_{v}-\gamma^{2} \mu_{2}
\end{array}\right]\left[\begin{array}{c}
\dot{u} \\
\dot{v}
\end{array}\right] .
$$

So, we have modified Jacobian matrix

$$
J=\left[\begin{array}{cc}
f_{u}-\gamma^{2} \mu_{1} & f_{v} \\
g_{u} & g_{v}-\gamma^{2} \mu_{2}
\end{array}\right] .
$$

For stability, there are two conditions such that $\operatorname{det}(J)>0$ and $\operatorname{tr}(J)<0$. Trace of the Jacobian matix $f_{u}+g_{v}-\gamma^{2}\left(\mu_{1}+\mu_{2}\right)<0$ and determinant $\left(f_{u}-\gamma^{2} \mu_{1}\right)\left(g_{v}-\gamma^{2} \mu_{2}\right)-f_{v} g_{u}>0$.

In the absence of diffusion, the stationary state solution is stable, and presence of diffusion, the solution becomes unstable. Let us consider $\mu_{1}=\mu_{2}=0$, the initial conditions for diffusion-driven instability easily seen

$$
\begin{aligned}
\operatorname{tr}(J) & =f_{u}+g_{v}<0, \\
\operatorname{det}(J) & =f_{u} g_{v}-f_{v} g_{u}>0 .
\end{aligned}
$$

Now consider the determinant,

$$
\begin{aligned}
\operatorname{det}(J) & =\left(f_{u}-\gamma^{2} \mu_{1}\right)\left(g_{v}-\gamma^{2} \mu_{2}\right)-f_{v} g_{u} \\
& =f_{u} g_{v}-\gamma^{2} f_{u} \mu_{2}-\gamma^{2} \mu_{1} g_{v}+\gamma^{4} \mu_{1} \mu_{2}-f_{v} g_{u} \\
& =\gamma^{4} \mu_{1} \mu_{2}-\gamma^{2}\left(\mu_{1} g_{v}+\mu_{2} f_{u}\right)+f_{u} g_{v}-f_{v} g_{u} .
\end{aligned}
$$

Instability will occur when

$$
\gamma^{4} \mu_{1} \mu_{2}-\gamma^{2}\left(\mu_{1} g_{v}+\mu_{2} f_{u}\right)+f_{u} g_{v}-f_{v} g_{u}<0 .
$$

Above inequality is quadratic in $\gamma^{2}$ and this is a parabola which opens up since $\mu_{1} \mu_{2}$ is positive. Getting the minimum value of the $\operatorname{det}(J)$, consider the derivative concerning $\gamma^{2}$ equal to zero

$$
\begin{aligned}
& \frac{d}{d \gamma^{2}}(\operatorname{det}(J))=2 \mu_{1} \mu_{2} \gamma^{2}-\left(f_{u} \mu_{2}+g_{v} \mu_{1}\right)=0 \\
\Rightarrow & \gamma^{2}=\frac{f_{u} \mu_{2}+g_{v} \mu_{1}}{2 \mu_{1} \mu_{2}} .
\end{aligned}
$$

To find the minimum value, set the above expression for $\gamma^{2}$ into the $\operatorname{det}(J)$, which is the third condition needed for diffusion instability to happen.

$$
\begin{aligned}
& \mu_{1} \mu_{2}\left(\frac{f_{u} \mu_{2}+g_{v} \mu_{1}}{2 \mu_{1} \mu_{2}}\right)^{2}-\left(f_{u} \mu_{2}+g_{v} \mu_{1}\right)\left(\frac{f_{u} \mu_{2}+g_{v} \mu_{1}}{2 \mu_{1} \mu_{2}}\right)+f_{u} g_{v}-f_{v} g_{u}<0 \\
\Rightarrow & \mu_{1} \mu_{2}\left(\frac{f_{u} \mu_{2}+g_{v} \mu_{1}}{2 \mu_{1} \mu_{2}}\right)^{2}-2 \mu_{1} \mu_{2}\left(\frac{f_{u} \mu_{2}+g_{v} \mu_{1}}{2 \mu_{1} \mu_{2}}\right)^{2}+f_{u} g_{v}-f_{v} g_{u}<0 \\
\Rightarrow & -\mu_{1} \mu_{2}\left(\frac{f_{u} \mu_{2}+g_{v} \mu_{1}}{2 \mu_{1} \mu_{2}}\right)^{2}+f_{u} g_{v}-f_{v} g_{u}<0 \\
\Rightarrow & \left(f_{u} \mu_{2}+g_{v} \mu_{1}\right)^{2}>4 \mu_{1} \mu_{2}\left(f_{u} g_{v}-f_{v} g_{u}\right) .
\end{aligned}
$$

Because of second condition required for instability $\left(f_{u} g_{v}-f_{v} g_{u}>0\right)$, it follows that

$$
f_{u} \mu_{2}+g_{v} \mu_{1}>2 \sqrt{\mu_{1} \mu_{2}\left(f_{u} g_{v}-f_{v} g_{u}\right)} .
$$

This further implies

$$
f_{u} \mu_{2}+g_{v} \mu_{1}>0
$$

The following four cases represent when diffusion instability happens 
i. $\operatorname{tr}(J)=f_{u}+g_{v}<0$

ii. $\operatorname{det}(J)=f_{u} g_{v}-f_{v} g_{u}>0$

iii. $f_{u} \mu_{2}+g_{v} \mu_{1}>0$

iv. $f_{u} \mu_{2}+g_{v} \mu_{1}>2 \sqrt{\mu_{1} \mu_{2}\left(f_{u} g_{v}-f_{v} g_{u}\right)}$

\section{LINEAR STABILITY ANALYSIS OF BRUSSELATOR MODEL}

Prigogine and his collaborators proposed a theoretical model, a Brusselator model for a type of autocatalytic reaction. This is undoubtedly the most straightforward system exhibiting Turing patterns $[9,10]$. Let $\alpha>0 ; \beta>0$ be given positive real numbers. In a finite domain $\Omega$, we consider the following system with a Neumann boundary condition

$$
\begin{cases}\frac{\partial u}{\partial t}=f(t, x, u, v)+\mu_{1} \Delta u, & t>0, x \in \Omega, \\ \frac{\partial v}{\partial t}=g(t, x, u, v)+\mu_{2} \Delta v, & t>0, x \in \Omega,\end{cases}
$$

where, $f(t, x, u, v)=\alpha-(\beta+1) u+u^{2} v$ and $g(t, x, u, v)=\beta u-u^{2} v$. Here, $f_{u}=-(\beta+1)+2 u v$, $f_{v}=u^{2}, g_{u}=\beta-2 u v$, and $g_{v}=-u^{2}$. Since there is diffusion part, so cases for which diffusion-driven instability will occur
i. $f_{u}+g_{v}=-(\beta+1)+2 u v-u^{2}<0$
ii. $u^{2}(\beta+1)+4 u^{3} v-\beta u^{2}>0$
iii. $f_{u} \mu_{2}+g_{v} \mu_{1}>0$
iv. $f_{u} \mu_{2}+g_{v} \mu_{1}>2 \sqrt{\mu_{1} \mu_{2}\left(f_{u} g_{v}-f_{v} g_{u}\right)}$

Here, we assume a specific two-species reaction diffusion model and carry out the whole study. We study the simplest reaction-diffusion mechanism, the uniform positive steady state $\left(u_{s}, v_{s}\right)$ is $f(t, x, u, v)=0$ and $g(t, x, u, v)=0$. Therefore,

$$
\begin{aligned}
& \alpha-(\beta+1) u+u^{2} v=0, \\
& \beta u-u^{2} v=0 .
\end{aligned}
$$

Solving these equations we get, $u_{s}=\alpha$ and $v_{s}=\frac{\beta}{\alpha}$, where $\alpha>0$ and $\beta>0$. At steady state

$$
\begin{aligned}
& f_{u}=-(\beta+1)+2 u_{s} v_{s}=\beta-1 \\
& f_{v}=u_{s}^{2}=\alpha^{2}>0 \\
& g_{u}=\beta-2 u_{s} v_{s}=\beta-2 \alpha \frac{\beta}{\alpha}=\beta-2 \beta=-\beta<0 \\
& g_{v}=-u_{s}^{2}=-\alpha^{2}<0 .
\end{aligned}
$$

Now, $f_{u} g_{v}-f_{v} g_{u}=(\beta-1)\left(-\alpha^{2}\right)-\alpha^{2}(-\beta)=-\alpha^{2} \beta+\alpha^{2}+\alpha^{2} \beta=\alpha^{2}>0$. Since $f_{u}$ and $g_{v}$ must have opposite signs we must have $\beta>1$. Using these, the following four cases will happen

i. $f_{u}+g_{v}=\beta-1-\alpha^{2}<0 \Rightarrow \beta-1<\alpha^{2}$

ii. $f_{u} g_{v}-f_{v} g_{u}=\alpha^{2}>0$

iii. Let $d=\frac{\mu_{2}}{\mu_{1}}$, then

$$
\begin{aligned}
& d f_{u}+g_{v}>0 \\
\Rightarrow & d(\beta-1)-\alpha^{2}>0 \\
\Rightarrow & d(\beta-1)>\alpha^{2}
\end{aligned}
$$


iv. $\left(d f_{u}+g_{v}\right)^{2}-4 d\left(f_{u} g_{v}-f_{v} g_{u}\right)>0$.

which yields $(d(\beta-1)-\alpha)^{2}>4 d \alpha^{2}$.

These inequalities define a domain in $(\alpha, \beta, d)$ parameter space, known as the Turing space (or pattern formation space).

\section{HOPF BIFURCATION}

Poincaree-Andronov-Hopf bifurcation or Hopf bifurcation arises since two eigenvalues cross the imaginary axis due to variation of the system of parameters. Mathematically, Hopf bifurcation is a critical coordinate where a system's stability alters and periodic result arises [11].

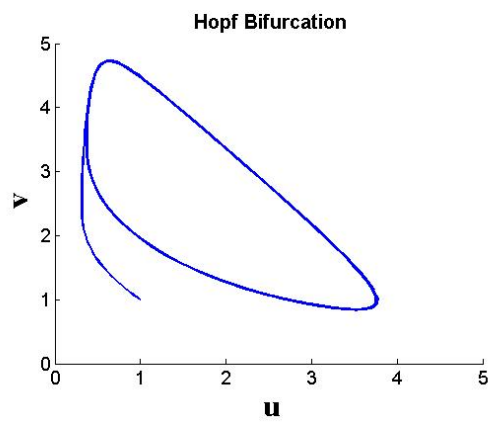

Fig. 1. Limit cycle of (1.1) using $\alpha=1$ and $\beta=3$ and maximum time is $\mathbf{1 2 0}$

The following figures show the limit cycle of the Brusselator model for different values of time along with parameters value $\alpha=1$ and $\beta=3$. The output of the program is given below when the maximum time is 120 (Fig. 1).
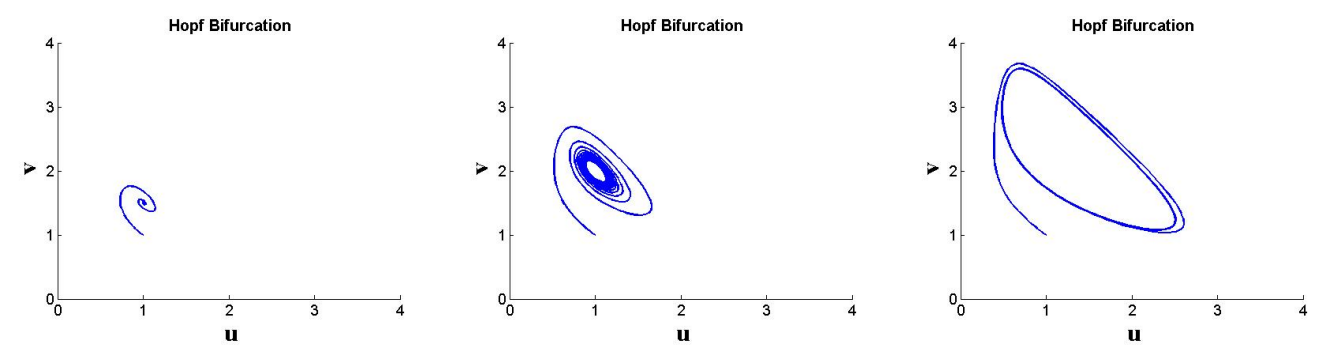

Fig. 2. Limit cycle of (1.1) using $\alpha=1$ and $\beta=3$ and when maximum time are 60,80 and 100 respectively

In Fig. 2, the limit cycles of the Brusselator model at different times are presented, mainly when time is 60,80 , and 100 , respectively.

By keeping all the parameters constant of reaction-diffusion equations except one, we can get the different stability conditions. So, by constructing a simple stability diagram, we can see the stability of the constant solution and bifurcation coordinates. 


\section{EXAMPLES AND APPLICA- TIONS}

In this section, we discuss the numerical results of the model. Positivity is one of the characteristics which occupy by the continuous systems. For example, the concentration of two reactant species represents unknown quantities in the Brusselator model. Moreover, a negative result of concentration from any numerical method becomes meaningless. For justifying unconditional stability and consis-tency of the mention finite difference method, we need to use Taylor series expansion, and Von Neumann stability scheme [12]. The model has been solved numerically using the explicit central finite difference method. Furthermore, as our considered model is an initial boundary value problem, we have used different types of periodic initial conditions.
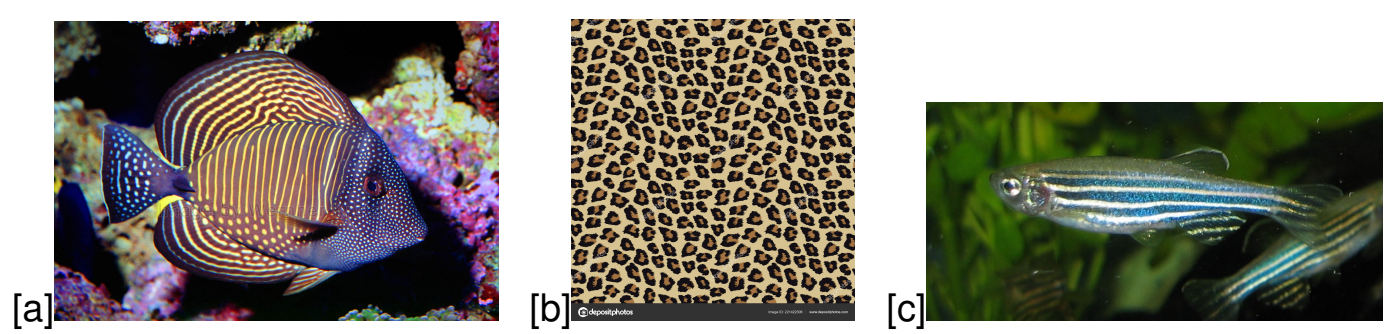

Fig. 3. (a) Sailfin Tang fish, (b) Skin pattern of Leopard, and (c) Zebra fish (Danio Rerio)

[a]

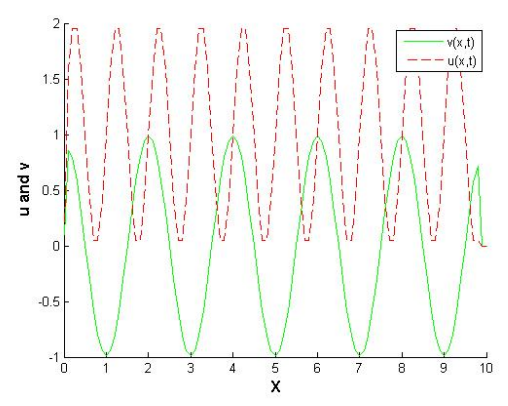

[b]
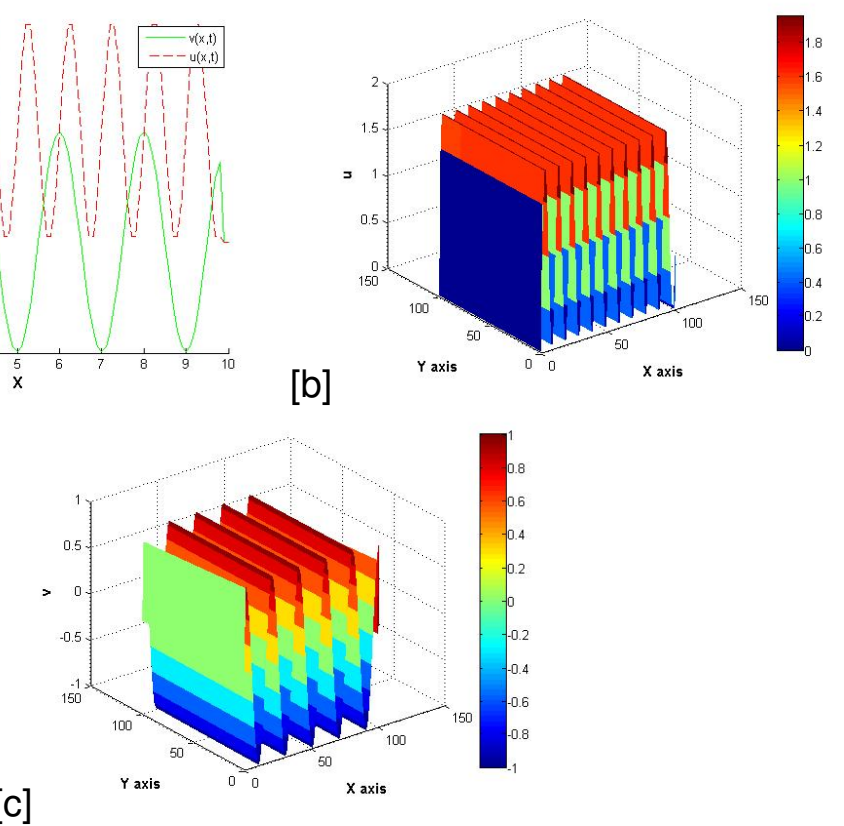

Fig. 4. Solutions for $\alpha=1, \beta=1.88, \mu_{1}=0.25$ and $\mu_{2}=10$ with initial conditions $u(0, x)=1+\sin (2 \pi x)$ and $v(0, x)=\cos (\pi x)$ where (a) Concentration of activator and inhibitor in 1D, (b) for $u$, and (c) for $v$ 
Here, pattern formation looks in the head of Sailfin Tang fish, spotted, the skin pattern of Leopard, which is also spotted, and skin of Zebrafish (Danio Rerio) which pattern formation is striped.

We want to analyze the behavior of the Brusselator model in two-dimensional coordinates, and we have seen that we have different types of patterns like spot or stripe depending on the parameter's values and initial conditions. To get such a pattern, we have used a 2D finite difference method. Here, we have used the Matlab program to simulate the result of the Brusselator model. We have seen from the simulations that we can get different natural patterns by the Brusselator model. If we change the parameter's value or initial condition, we will get different colors and design patterns. However, we can not change the value simultaneously. We have to follow the stability conditions. If those four stability conditions (described in section 3) are satisfied, and there are good initial conditions, then we will get the different patterns.

There are several patterns of formation seen in living life. Some are spot, or some are stripe, but their pattern formation is always different from individuals. The following figure shows some pattern formation in the skin of animals.

Example 1. The output of this model, when the values of the parameters are $\alpha=1, \beta=1.88$, $\mu_{1}=0.25$ and $\mu_{2}=10$ and the initial conditions are $u(0, x)=1+\sin (2 \pi x)$ and $v(0, x)=\cos (\pi x)$.

Example 2. The output of this model, when $\alpha=1, \beta=1.88, \mu_{1}=1, \mu_{2}=10$ and the initial condition $u(0, x, y)=-3+\cos x+\sin y-$ $7 \cos y \sin x$ and $v(0, x, y)=3$ is [a]
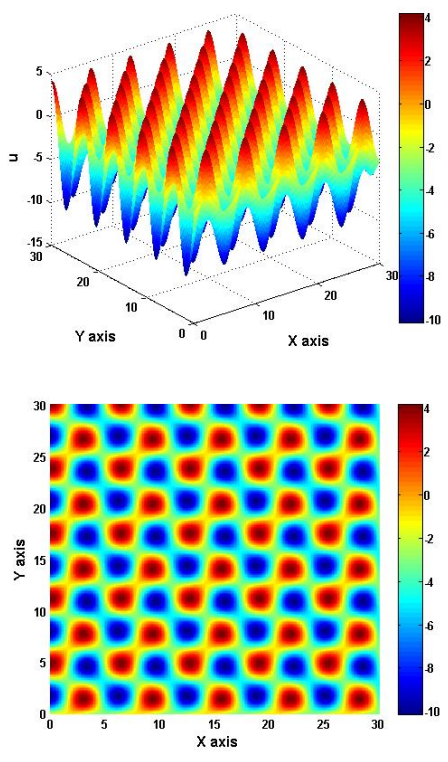

[b]

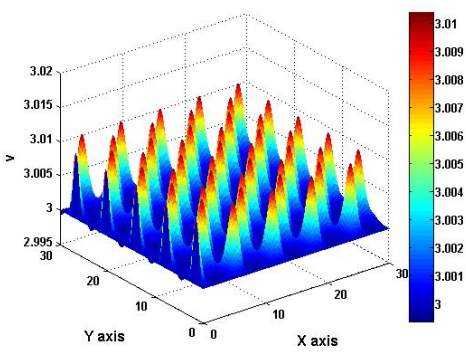

[d]

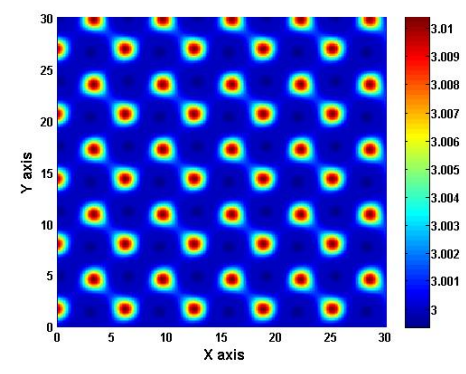

Fig. 5. Pattern solutions for $\alpha=1, \beta=1.88, \mu_{1}=1$ and $\mu_{2}=10$ with $u(0, x, y)=-3+\cos x+\sin y-7 \cos y \sin x$ and $v(0, x, y)=3$ while (a) and (b) represent the surface plot of concentration of activator and inhibitor, (c) and (d) represent the view from top of (a) and (b)

In nature, we can get animal's or bird's pattern like the outputs. The pattern on the head of Sailfin Tang fish (Fig. 3(a)) is similar to the pattern in Fig. 5(c,d). 
[a]
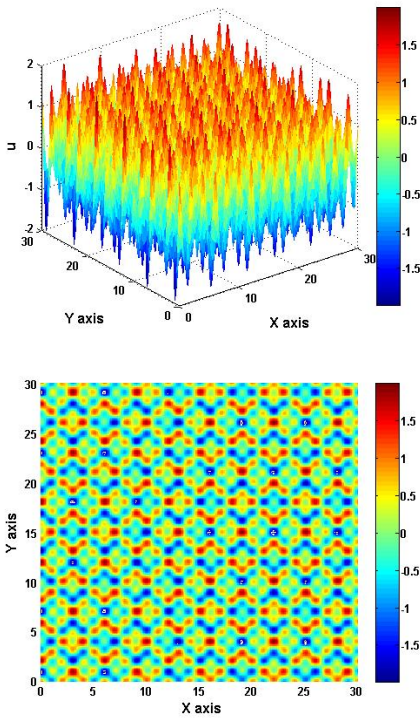

[b]

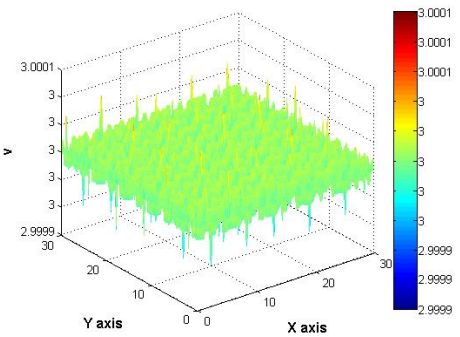

[d]

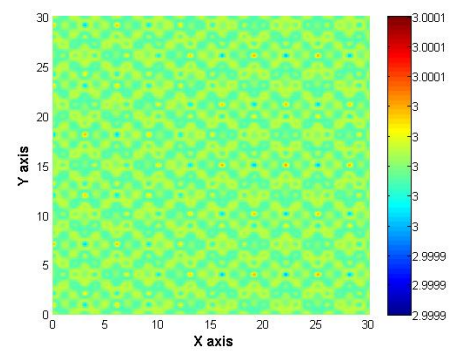

Fig. 6. Pattern formations for $\alpha=1, \beta=1.8, \mu_{1}=0.25$ and $\mu_{2}=12$ with $u(0, x, y)=-\sin (2 x) \cos (y)+\cos (\pi x) \cos (\pi y)$ and $v(0, x, y)=3$ where (a) and (b) represent the surface plot of concentration of activator and inhibitor, (c) and (d) represent the view from top of (a) and (b)

[a]
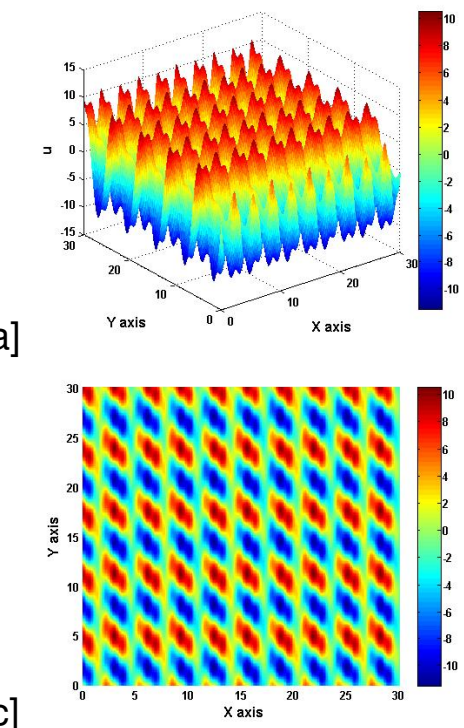

[b]

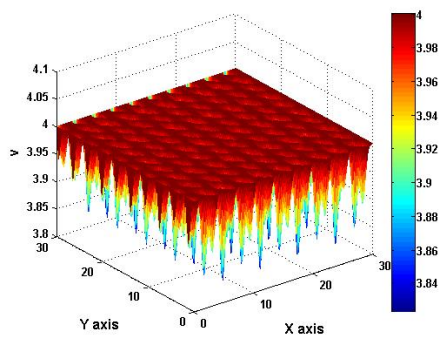

[d]

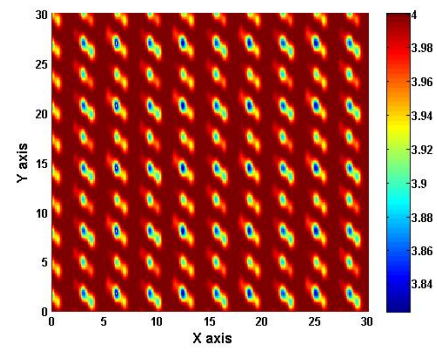

Fig. 7. Patterns for $\alpha=1, \beta=1.88, \mu_{1}=0.25$ and $\mu_{2}=12$ with

$u(0, x, y)=-0.5+\cos (x-6 y)+\sin y-10 \cos y \sin (x+y)$ and $v(0, x, y)=4$ where (a) and (b) represent the surface plot of concentration of activator and inhibitor, (c) and (d) represent the view from top of (a) and (b) 
[a]

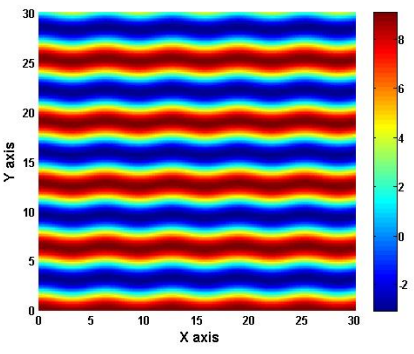

[b]

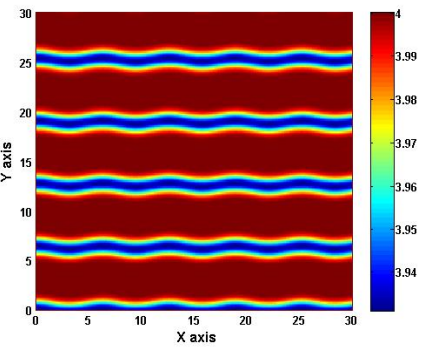

Fig. 8. Pattern solutions for $\alpha=1, \beta=1.88, \mu_{1}=1$ and $\mu_{2}=10$ with $u(0, x, y)=3+\sin x \cos y+6 \cos x$ and $v(0, x, y)=4$ where (a) and (b) represent the top of activator and inhibitor

Example 3. Now, by changing the value of parameter to $\alpha=1, \beta=1.8, \mu_{1}=$ 0.25 and $\mu_{2}=12$ and initial conditions to $u(0, x, y)=-\sin (2 x) \cos (y)+\cos (\pi x) \cos (\pi y)$ and $v(0, x, y)=3$, we get this following patterns.

Sometimes, the pattern of veiled chameleon and grasshopper is likely similar to the output pattern. In our nature, peacocks, butterflies, flowers, birds, and animals combine different colors and design patterns.

Example 4. Again we will change value's of parameters and both of the initial conditions such that $\alpha=1, \beta=1.88, \mu_{1}=0.25$ and $\mu_{2}=12$ and $u(0, x, y)=-0.5+\cos (x-6 y)+$ $\sin y-10 \cos y \sin (x+y)$ and $v(0, x, y)=4$ to get following patterns. The pattern on the skin of Leopard is lightly similar to the pattern of Fig. 7(c,d) and one can compare with Fig. 3(b).

Example 5. Finally, in the last example, we also can get the strip pattern by changing the value's of the parameters to $\alpha=1, \beta=1.88$, $\mu_{1}=1$ and $\mu_{2}=10$ and the initial conditions to $u(0, x, y)=3+\sin x \cos y+6 \cos x$ and $v(0, x, y)=4$. Some kinds of zebra fishes such as Danio Rerio, Rerio x dangila, Rerio x kyathit, Rerio $x$ kerri has stripe pattern similar to the pattern in Fig. 8(a,b); check the pattern using Fig. 3(c).

Nature is a combination of different color patterns. In the above, we see that the output of the Brusselator model gives such a pattern. These various patterns come from changing the value of parameters and initial conditions of the Brusselator model. There is also the effect of geometry. According to the initial condition and geometry, the pattern will be stripe or spot. Nevertheless, we cannot take the harmful concentration because it is meaningless. The parameter's value should satisfy the stability condition, and the initial condition should be valid for getting a pattern. It is remarked that the stable results found in application since the theoretical stable condition are satisfied according to the choice of parametric values; see the theoretical results as presented in Section 2 and 3.

\section{CONCLUSION}

This paper has illustrated qualitative testimony in backing a spatial and time-varying problem for pattern formation, analogous with living life and inert objects of multiple species. Delineating this, we choose the Brusselator model and solve this model by applying a finite difference scheme for various values of the parameters and initial conditions with Neumann boundary conditions. Moreover, we have seen that initial conditions have a significant influence on pattern formation. From the reasoning study, we get a conspicuous idea about the mechanism of two species in pattern formation by the Brusselator model in a spatially heterogeneous environment. Theoretical observations and numerical simulations clinch our assumption, which is also realistic. In a nutshell, different pattern formations can generate by the impact of geometry, various parameter values, and several 
initial conditions from the Brusselator model, which is analogous with spot or stripe with reallife objects.

\section{ACKNOWLEDGEMENT}

The authors are acknowledged to the anonymous reviewers for their constructive suggestions to improve the quality of the manuscript significantly. The author M. Kamrujjaman research was partially supported by TWAS grant 2019_19-169 RG/MATHS/AS_I.

\section{COMPETING INTERESTS}

Authors have declared that no competing interests exist.

\section{REFERENCES}

[1] Hossine Z, Kamrujjaman M. On the Interplay of Geometrical Shapes and the Analysis of a Dispersal Model for Pattern Formations. J. Adv. Math. \& Comp. Sci. 2019;34(4):1-10.

[2] Deutsch A, Dormann S. Cellular Automaton Modeling of Biological Pattern Formation. Springer Science and Business Media; 1960.

[3] Ball P. The self-made tapestry: Pattern formation in nature. Oxford University Press; 2001.
[4] Stoltz, Marnus S. Pattern formation in the Brusselator model of chemical reactions. PhD dissertation, University of Pretoria; 2016.

[5] Murray JD. Spatial Models and Biomedical Applications. Math. biol., Springer-Verlag, Berlin; 2003.

[6] Grindrod P. The theory and applications of reaction-diffusion equations. Oxford University Press; 1996.

[7] Arcuri P, Murray JD. Pattern sensitivity to boundary and initial conditions in reaction-diffusion models. J. Math. Biol. 1986;24(2):141165.

[8] Garcia A, Alexandra L. Reaction diffusion systems and pattern formation. University of Barcelona; 2016.

[9] Murray JD. Mathematical Biology. Berlin; New York: Springer-Verlag; 1989.

[10] Catalano G, Eilbeck JC, Monroy A, Parisi E. A mathematical model for pattern formation in biological systems. Physica D:Nonlinear Phenomena. 1981;3(3):439-456.

[11] Brown KJ, Eilbeck JC. Bifurcation, stability diagrams, and varying diffusion coefficients in reaction-diffusion equations. Bulletin Math. Biol. 1982;44(1):87-102.

[12] Ames WF. Numerical method for partial differential equations. New York, Academic press; 1977. 


\section{A APPENDIX}

If we have no diffusion, the concentration of $u$ and $v$ change due to reaction

$$
\begin{aligned}
& \frac{\partial u}{\partial t}=f(t, x, u, v), \\
& \frac{\partial v}{\partial t}=g(t, x, u, v),
\end{aligned}
$$

where $f$ and $g$ represent the reaction rate expression. Now, steady state is given by

$$
\begin{aligned}
& f\left(u^{*}, v^{*}\right)=0 \\
& g\left(u^{*}, v^{*}\right)=0 .
\end{aligned}
$$

It is spatially uniform. Assume this is a stable stationary state solution and the corresponding Jacobian matrix is

$$
J=\left(\begin{array}{ll}
f_{u} & f_{v} \\
g_{u} & g_{v}
\end{array}\right) .
$$

Here, $f_{u}$ represents the derivative of $f$ with respect to $u$ and $f_{v}$ represents the derivative of $f$ with respect to $v$. Similarly, $g_{u}$ and $g_{v}$ have the similar meaning. We can get the eigenvalues $(\lambda)$ from the system by

$$
|J-\lambda \mathcal{I}|=0
$$

where, $\mathcal{I}$ is an $2 \times 2$ identity matrix; which gives,

$$
\begin{aligned}
& \left(f_{u}-\lambda\right)\left(g_{v}-\lambda\right)-f_{v} g_{u}=0 \\
\Rightarrow & f_{u} g_{v}-f_{u} \lambda-\lambda g_{v}+\lambda^{2}-f_{v} g_{u}=0 \\
\Rightarrow & \lambda^{2}-\left(f_{u}+g_{v}\right) \lambda+\left(f_{u} g_{v}-f_{v} g_{u}\right)=0 .
\end{aligned}
$$

For stability, we need to satisfy two conditions, such that $\operatorname{det}(J)>0$ and $\operatorname{tr}(J)<0$. So, from here we get $[1,5]$,

$$
\begin{aligned}
& f_{u}+g_{v}<0, \\
& f_{u} g_{v}-f_{v} g_{u}>0 .
\end{aligned}
$$

(C) 2021 Hossine et al.; This is an Open Access article distributed under the terms of the Creative Commons Attribution License (http://creativecommons.org/licenses/by/4.0), which permits unrestricted use, distribution, and reproduction in any medium, provided the original work is properly cited.

\section{Peer-review history:}

The peer review history for this paper can be accessed here: http://www.sdiarticle4.com/review-history/69331 